\title{
Weight Change
}

National Cancer Institute

\section{Source}

National Cancer Institute. Weight Change. NCI Thesaurus. Code C9232.

An increase or decrease in the weight of an individual. 